their mouths reflections of marvellous candour.

In the beginning was Geoffrey Harris, the English physiologist, who first postulated that the brain directs the release of pituitary hormones by despatching chemical messages from the hypothalamus to the pituitary. Guillemin and the Canadian endocrinologist, Murray Saffran, with whom Schally was then working as a technician, obtained early and independent evidence of the existence of such factors, and there ensued an uneasy collaboration between Schally and Guillemin that soon gave place to an ungovernable rivalry. According to The Devil's Dictionary calamities are of two kinds; misfortune to ourselves and good fortune to others. Guillemin and Schally dogged each others' footsteps through success and failure, each cast down by the other's victories and elated at his defeats; when, for example, Schally's group took for a releasing factor a proteolytic fragment of myoglobin, which they laboriously isolated and sequenced after processing a quarter of a million brains, Guillemin did not conceal his pleasure.

In the laboratory it seems that both Guillemin and Schally (though both had proved their ability as young men) made only minor contributions to the work of their groups, but it was they who kept their groups in being. The burden of the science was borne by a superlatively capable corps of young men - Burgus and Vale, Ward and Brazeau for Guillemin; the Japanese, Arimura, Matsuo and Baba, together with Radding, Carter and Nair for Schally. Their efforts were ill-rewarded - indeed, on the evidence here, it is small wonder that a postdoctoral liberation movement of a kind appears to be emerging in the United States.

Both teams had remarkable achievements. One that stands out is a dazzling feat of peptide chemistry by the Japanese visitor, Matsuo, who determined the sequence of LRF by simultaneous analysis of unfractionated, overlapping proteolytic fragments on $50 \mu \mathrm{g}$ of material, and so won the race for Schally.

The Nobel Duel is a hugely entertaining read, but it is also much more, for it lays bare the mechanics of Big Science (into which biology is being increasingly assimilated), and exposes its virtues and blemishes to public view. Whether the process of discovery feeds on such ferocious competitiveness as is here on display is an interesting and indeed important question. Wade opines that other highly regarded workers in the field, such as Saffran and Hearn, had too much of a

The second volume of Colin Ronan's The Shorter Science \& Civilisation in China has just been published by Cambridge University Press. The book is an abridgement of the material in Vols III and IV/I (for reviews see Nature 186, 36 and 196, 844) of Joseph Needham's original text, and costs $£ 15$. sense of humour and of the fitness of things to engage in such destructive proceedings; this fastidiousness, just as a classical education has been alleged to do, enabled them perhaps to despise the success that it prevented them from achieving. Nonetheless, it is remarkable how close other laboratories with vastly smaller commitments and funds, such as that of Harris, came to a solution of the problem.

A piquant footnote to the history of the long haul to the structure and function of the releasing factors is the reaction of an early adversary of Harris's, that most entrenched of all the illuminati of the scientific establishment, Lord (Solly) Zuckerman. He, it seems, remains adamant that Harris was wrong from the outset and that the pituitary hormone releasing factors are, after all, no more than mirage.

Walter Gratzer is in the MRC Cell Biophysics Unit, King's College, University of London.

\title{
Mysteries of the zeta potential examined
}

\section{John Gregory}

Zeta Potential in Colloid Science: Theory and Application. By Robert J. Hunter. Pp.386. ISBN 0-12-361960-2. (Academic Press: 1981.) $£ 35, \$ 84$.

ELECTROKINETIC phenomena, such as streaming potential and electrophoresis, have been known for over a century. They arise whenever there is relative motion between a charged interface and a liquid and are usually interpreted in terms of the zeta potential, which is conventionally defined as the electric potential at the plane of shear. There has always been an air of mystery surrounding the concept of zeta potential, partly because of theoretical problems concerning the structure of the electrical double layer and the precise location of the shear plane within it, and partly because of difficulties in obtaining reliable experimental values. Although there have been several books on electrokinetic phenomena generally, Professor Hunter's is the only one, to my knowledge, which is devoted to the enigmatic zeta potential itself.

To a large extent, the book is successful in drawing together and rationalizing a vast amount of material covering many aspects of the subiect. It should be essential reading for those embarking on research in the area and most established practitioners will want to have a copy within reach, or at least in the library of their institution.

After a brief, scene-setting introduction, the author first deals with the distribution of charge and potential at interfaces. Most of this material is readily available elsewhere and the treatment here offers no new insights. However, since it forms the basis of much of what follows, it could not reasonably have been left out.

By far the most useful chapters are those concerned with experimental techniques and the calculation of zeta potentials. There is an exceptionally clear and up-todate exposition of the procedures for obtaining zeta potentials from electrophoretic mobilities. The experimental chapter includes a wealth of practical detail, such as the design of electrophoresis cells, and even advice on how to avoid leakage problems with platinum streaming potential electrodes.

The chapter on electroviscous and viscoelectric effects is an admirable survey, a task which does not seem to have been previously attempted. Hunter's conclusion from the discussion of viscoelectric effects is that the electrokinetic plane of shear cannot lie too far from the outer Helmholtz plane and that the zeta potential must be nearly equal to the potential just inside the diffuse layer. This will comfort the many colloid scientists who have been making similar assumptions for years; but those who have advocated comparatively thick layers of structured water at interfaces may still remain unconvinced.

The remainder of the book is concerned with applications of zeta potential and the effect of simple inorganic ions and more complex adsorbates. The discussion of oxide surfaces raises the important question of the distinction between points of zero charge and isoelectric points, but, unfortunately, in an uncharacteristic lapse from his usually clear style, the author creates some confusion, especially with regard to the role of specifically adsorbed ions. There are mentions of applications in such areas as colloid stability, flotation and electrophoretic deposition, but they are too briet to do justice to these various subjects.

It becomes evident from the final chapters that there are still major uncertainties concerning the nature of the electrical double layer at solid-liquid interfaces. In some cases, experimental values of charge density and zeta potential can only be reconciled with current models by making rather unrealistic assumptions, as the author readily admits. Perhaps it is time for a thorough reappraisal of concepts such as the Stern layer and molecular capacitors, bearing in mind that on this scale $(c .1 \mathrm{~nm})$ water is not a structureless medium and that real surfaces are almost never smooth.

John Gregory is Reader in Water Chemistry in the Department of Civil Engineering, University College, University of London, and Secretary of the Colloid and Surface Chemistry Group, Society of Chemical Industry. 